\title{
Fractal Dimension of Backbone of Eden Trees
}

\author{
S. S. Manna ${ }^{1}$ and D. Dhar ${ }^{2}$ \\ ${ }^{1}$ Department of Physics, Indian Institute of Technology \\ Powai, Mumbai 400076, India \\ e-mail : manna@niharika.phy.iitb.ernet.in \\ ${ }^{2}$ Theoretical Physics Group, Tata Institute of Fundamental Research \\ Homi Bhabha Road, Mumbai 400005, India \\ e-mail : ddhar@theory.tifr.res.in
}

\begin{abstract}
$\underline{\text { Abstract }}$
We relate the fractal dimension of the backbone, and the spectral dimension of Eden trees to the dynamical exponent $z$. In two dimensions, it gives fractal dimension of backbone equal to $4 / 3$ and spectral dimension of trees equal to $5 / 4$. In three dimensions, it provides us a new way to estimate $z$ numerically. We get $z=1.617 \pm 0.004$.
\end{abstract}

Dense branching patterns are found in many different physical situations in nature e.g., coral reefs, river networks, collapsed phase of branched polymers, very slowly evaporated films of sugar dissolved in water [1-3]. In all these systems, the Hausdorff dimension of the structure is equal to that of the embedding space but the detailed structure is different depending on the different physical processes involved.

The Eden model has been studied a lot in the last decade, mainly for the surface properties [4]. Eden trees [5,6] are simple theoretical model of dense branching structures. In [5], it was argued that classical diffusion on Eden trees is anomalous because of trapping in dead end branches, and the root mean square deviation of a random walker on the tree increases with time as $t^{x}$, where the exponent $x$ does not satisfy the usual relation $x=\tilde{d} / 2 \bar{d}$, where $\tilde{d}$ is the spectral dimension, and $\bar{d}$ is the (Hausdorff) fractal dimension of the lattice. Dhar and Ramaswamy expressed the exponents $x$ and $\tilde{d}$ in terms of an exponent $\theta$ related to the fractal dimension of the backbone of the trees [5]. Using a different method of analysis, and somewhat larger simulations, Nakanishi and Herrmann [6] also calculated these exponents. However, these exponents have not been determined analytically so far.

In this Rapid Communication, we show that the backbone exponent of the Eden trees can be related to the dynamical exponent of KPZ model, and show that in two dimensions, $\theta=1 / 3, \tilde{d}=5 / 4$ and $x=3 / 8$. In three dimensions, our method gives us a new way to determine the dynamical exponent numerically. The numerical determined value is $z=1.617 \pm 0.004$.

We shall consider Bond-Eden Trees (BET) in this report. The model is defined in 
terms of the spreading of an infectious disease along the bonds of a $d$-dimensional lattice. Each site may be in one of two states : healthy or infected. At time $t=0$, a fixed set of 'seed' sites are infected. This seed set is a single site in the so-called point seed geometry, and a $(d-1)$ dimensional hyper-plane in layer seed geometry. At each time-step, we select at random one site from the set of healthy sites having at least one infected neighbour. This site then becomes infected, and once infected, a site never recovers. We connect this site to existing infected cluster by the bond connecting it to its first-infected neighbour. Each new infected site adds exactly 1 bond to the cluster. The resulting cluster so generated is called a BET.

However, the bulk of the Bond Eden trees is a spanning tree, and has a complex internal structure. This may be quantified in terms of the fractal dimension of the chemical paths along the tree, distribution of branch sizes, spectral dimension of the tree, structure of its backbone etc. We shall see below that all these measures can be determined in terms of a single critical exponent, which we choose to be the dynamical critical exponent $z$.

There is a unique path which connects any two sites of a tree. We define the backbone of the tree corresponding to radius $R$ as the set of all sites which lie on a path connecting one of the seed sites to any of the sites of the tree at a distance $R$ from it. Fig. 1 shows the backbone of a two dimensional Eden tree grown from a single seed for two values $R=80$ and 130 lattice spacings. As the cluster grows, some of the growing branches became dead ends, and are removed from the backbone. While the part of the backbone near the surface changes quite fast, structure of the backbone deep inside the cluster does not change much with time, and gets frozen in.

From Fig. 1, it is easy to see that the backbone of Eden clusters has a branching structure, where each branch is directed radially outwards. A branch of the backbone of length $R$ has transverse fluctuations of order $R^{1 / z}$, where clearly $z \geq 1$ and is called the dynamical exponent. This exponent is inverse of the exponent specifying the transverse fluctuations of a directed polymer in a random medium [7].

We now argue that the backbone has a fractal dimension $d_{B}$ given by

$$
d_{B}=1+(d-1)(1-1 / z)
$$

Consider the backbone of the cluster when its radius is $R$, and at a later stage when its radius is $R+h, h<<R$. Only a small fraction of the perimeter sites at radius $R$ remain part of the backbone at the later stage. Each such site gives rise to a cluster of active sites of transverse size $h^{1 / z}$. This implies that the density of backbone sites at radius $R$, when cluster has grown to size $(R+h)$ vanishes as $h^{-(d-1) / z}$ for large $h<<R$. Once $h$ is of order $R$, it gets frozen to the value $R^{-(d-1) / z}$, and does not change further. Thus the number of sites in the frozen backbone upto radius $R$ varies as $R^{d_{B}}$, where $d_{B}$ is given by Eq.(1).

The spectral dimension of the Eden trees is clearly a bulk property and can also be expressed in terms of $z$. Consider a spring network on the tree, such that at each bond of the tree, there is a spring of spring constant $\kappa$, and a mass $m$ is attached to each site of the tree. Let $F\left(\omega^{2}\right)$ be the fractional number of eigenvalues with frequency less than $\omega$. Then if $F\left(\omega^{2}\right) \sim \omega^{\tilde{d}}$ for small $\omega, \tilde{d}$ is the spectral dimension of the tree. 
To determine $\tilde{d}$, we use a decimation argument analogous to that in [8]. Define the 'burning time' at a site on the tree $T$ as the length of the longest directed path from the site to a leaf site of the tree. We construct a decimated tree $T^{\prime}$, consisting of only those sites whose burning time is an exact multiple of an integer scale factor $b$. The resulting tree $T^{\prime}$ is not related to $T$ by a simple scale change (for some figures of decimated trees see [9]). However, small parts of $T$ are related to corresponding parts of $T^{\prime}$ by local self-affine transformation, where radial distances are scaled by a factor $b$, and transverse distances by a factor $b^{1 / z}$. The spring constants renormalize by $\kappa^{\prime}=\kappa / b$, and masses according to the formula $M^{\prime}=b^{1+(d-1) / z} M$. Thus we get

$$
F\left(\kappa^{\prime} / M^{\prime}\right)=b^{-1-(d-1) / z} F(\kappa / M) .
$$

which implies that

$$
\tilde{d}=2 \cdot \frac{z+d-1}{2 z+d-1}
$$

For $d=2$, the dynamical exponent takes the well known KPZ value $3 / 2$. Correspondingly, we get $d_{B}=4 / 3$ and $\tilde{d}=5 / 4$. This value is in good agreement with the earlier numerical estimates of [5] and [6]. Dhar and Ramaswamy found that for $d=2$, $\tilde{d}=1.22 \pm 0.04, x=0.42 \pm 0.04$, and for $d=3, \tilde{d}=1.30 \pm 0.12, x=0.44 \pm 0.04[5]$. Nakanishi and Herrmann estimated $d=2, \tilde{d}=1.22 \pm 0.02$ and $x=0.39 \pm 0.02$ where as for $d=3, \tilde{d}=1.32 \pm 0.02$ and $x=0.30 \pm 0.02$ [6]. As $d \rightarrow \infty, \tilde{d}$ tends to 2 , which again agrees with the exact result [10].

For the root mean square displacement of order $L$ the average number of branch site per backbone site visited is of order $L^{(d-1) / z}$. Hence the diffusion constant is of order $L^{-(d-1) / z}$ and time $T$ scales as $L^{2+(d-1) / z}$, so we get $1 / x=2+(d-1) / z$. We note that $x \neq \tilde{d} / 2 \bar{d}$, where $\bar{d}$ is the fractal dimension of the substrate. However, the equality sign holds if $\bar{d}$ is replaced by $d_{m}=1+(d-1) / z$, which is the effective mass dimension of the graph defined by the relation that the number of distinct sites within a distance $r$ along the tree from a randomly chosen site (not near origin) varies as $r^{d_{m}}$ for large $r<<$ size of cluster $R$.

We have checked these predictions against numerical simulations. Figure 2 shows a plot of the average number of sites in the backbone $\left\langle M_{b}\right\rangle$ of a 2-dimensional Bond Eden tree within a (Euclidean) distance $R$ from the origin multiplied by a factor $R^{-4 / 3}$ versus $R^{-1 / 3}$. We averaged over 12 million independently generated configurations for $R=16$ decreased to 12000 configurations for $R=1024$ on the square lattice. We see a reasonably good fitting of a straight line in fair agreement with the theoretical prediction of $d_{B}=4 / 3$ which also indicates that the correction to scaling is likely to be $R^{-1 / 3}$. We estimate the error in the quoted values of both the fractal dimension and the correction to scaling exponent to be about 0.03 .

In Fig. 3 we show the results of simulations of Eden trees on a triangular lattice grown from a line seed on a $6000 \times 10000$ lattice, averaged over 2500 configurations. We have plotted $h^{2 / 3} N_{h}$ versus $h$, where $N_{h}$ is the fractional number of distinct trees which survive up to height $h$. From the scaling hypothesis $N_{h} \sim h^{-\alpha}$, where $\alpha=(d-1) / z$ In this case $\alpha=2 / 3$, and hence the graph should be a horizontal line. For large $h$ we 
see that this expectation is very well satisfied, and the numerically determined value of $z$ from this plot gives $z=3 / 2 \pm 0.002$.

The main advantage of our simulation over earlier simulations of various versions of Eden model, e.g., models A, B and C of Jullien and Botet [12] is that here we are in effect studying bulk quantities (The quantity $N_{h}$ can be related to the probability that randomly chosen site in the bulk of the tree has at least one descendant left after $h$ more generations). A related quantity, the distribution of branch sizes which are disconnected on removing a randomly chosen bond from the tree has been studied in [9].

Encouraged by the good convergence of simulation results in our model to the asymptotic values in 2-dimensions, we extended our studies to $2+1$ dimensions. In Fig. 4 , we plot the results of simulation of Eden growth in $2+1$ dimension in the layer geometry. We used as $180 \times 180 \times 500$ simple cubic lattice, and averaged over 10500 configurations. Fig. 4 shows $h^{5 / 4} N_{h}$ versus $h$ on a log-log plot. We estimate the slope of the curve in this plot to be $0.013 \pm .003$. This implies that $\alpha=5 / 4-0.013 \pm 0.003$, which corresponds to $z=1.617 \pm .004$. The value $\alpha=5 / 4$ corresponds to the Kim-Kosterlitz conjecture [13] which is clearly ruled out by our data. Our values are in good agreement with the current best numerical estimate of $\alpha=1.240 \pm 0.001$ by Forrest and Tang [14] and Ala-Nissila and Venalainen's result of $\alpha=1.240 \pm 0.002$ [15].

The extension to higher dimensions seems possible, but would require higher computational power than available to us at present.

We thank Drs. T. Halpin-Healy and L. H. Tang for providing some useful references. 


\section{Figure Captions}

Figure 1 : Backbone of a Bond Eden Tree generated on the square lattice from a point seed at two stages of growth of radii 80 and 130 lattice constants.

Figure 2 : Variation of average mass of the backbone $\left\langle M_{b}\right\rangle$ of Bond Eden Trees of radius $R$. The fractal dimension of the backbone is $\approx 4 / 3$, in complete agreement with our theoretical prediction and the correction to scaling decreases as $R^{-1 / 3}$.

Figure 3 : Plot of $h^{2 / 3} N_{h}$ versus $h$ for Bond Eden Trees grown on a base line of length 6000. Here $N_{h}$ is the fractional number of distinct trees which survive upto height $h$. The average slope of this plot for $h$ between 1000 and 10000 is at most \pm 0.003 .

Figure 4 :Plot of $h^{5 / 4} N_{h}$ versus $h$ for Bond Eden Trees grown on a square base of size $180 \times 180$. The average slope between $h=100$ and 500 is 0.013 . A straight line of slope 0.013 is plotted as a guide to the eye. 


\section{$\underline{\text { References }}$}

[1.] S. S. Manna and B. Subramanian, Phys. Rev. Lett. 76 (1996) 3460; A. Rinaldo, I. Rodriguez-Iturbe, R. Rigon, E. Ijjasz-Vasquez and R. L. Bras, Phys. Rev. Lett. 70, 822 (1993); A .Giacometti, A. Maritan, and J. R. Banavar, Phys. Rev. Lett. 75, 577 (1995).

[2.] B. Derrida and H. J. Herrmann, J. Phys. (Paris) 44 (1983) 1365.

[3.] A. S. Paranjape, Phys. Lett. A 176 (1993) 349.

[4.] For a review see P. Meakin, Phys. Rep. 235 (1993) 189; T. Halpin-Healy and Y. C. Zhang, Phys. Rep. 254 (1995) 215.

[5.] D. Dhar and R. Ramaswamy, Phys. Rev. Lett. 54, (1985) 1346.

[6.] H. Nakanishi and H. J. Herrmann, J. Phys. A 26 (1993) 4513.

[7.] S. Roux, A. Hansen and E. L. Hinrichsen, J. Phys. A 24, (1991) L295; M. Cieplak, A. Maritan and J. R. Banavar, Phys. Rev. Lett. 76, 3754 (1996).

[8.] S. S. Manna, D. Dhar and S. N. Majumdar, Phys. Rev. A 46 (1992) R4471.

[9.] P. Meakin, Physica Scripta 45 (1992) 733.

[10.] D. Dhar, J. Phys. A 18 (1985) L713.

[11.] J. Krug and P. Meakin, Phys. Rev. A 40 (1989) 2064.

[12.] R. Jullien and R. Botet J. Phys. A 18 (1985) 2279.

[13.] J. M. Kim and J. M. Kosterlitz, Phys. Rev. Lett. 62 (1989) 2289.

[14.] B. M. Forrest and L. H. Tang, Phys. Rev. Lett 64 (1990) 1405.

[15.] T. Ala-Nissila and O. Venalainen, J. Stat. Phys. 76 (1994) 1083. 


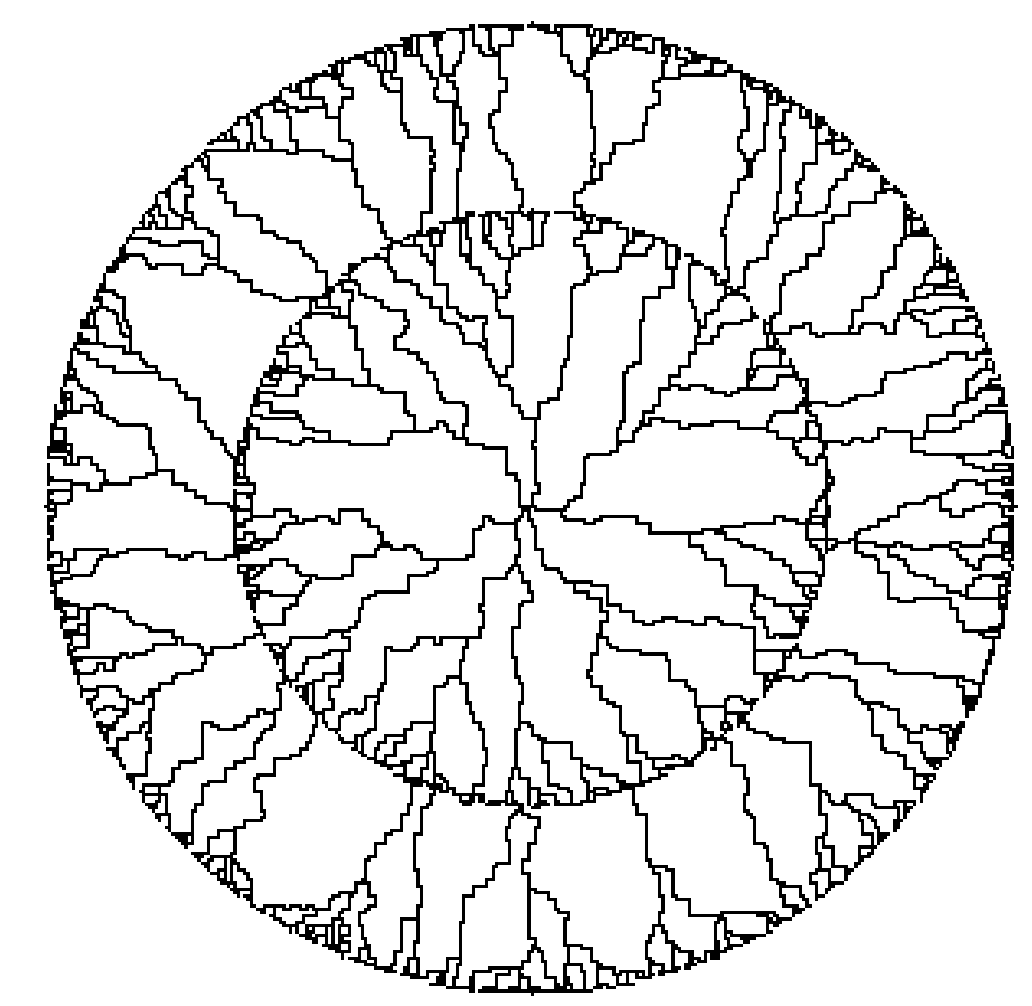

Fig 1 (Manna and Dhar) 


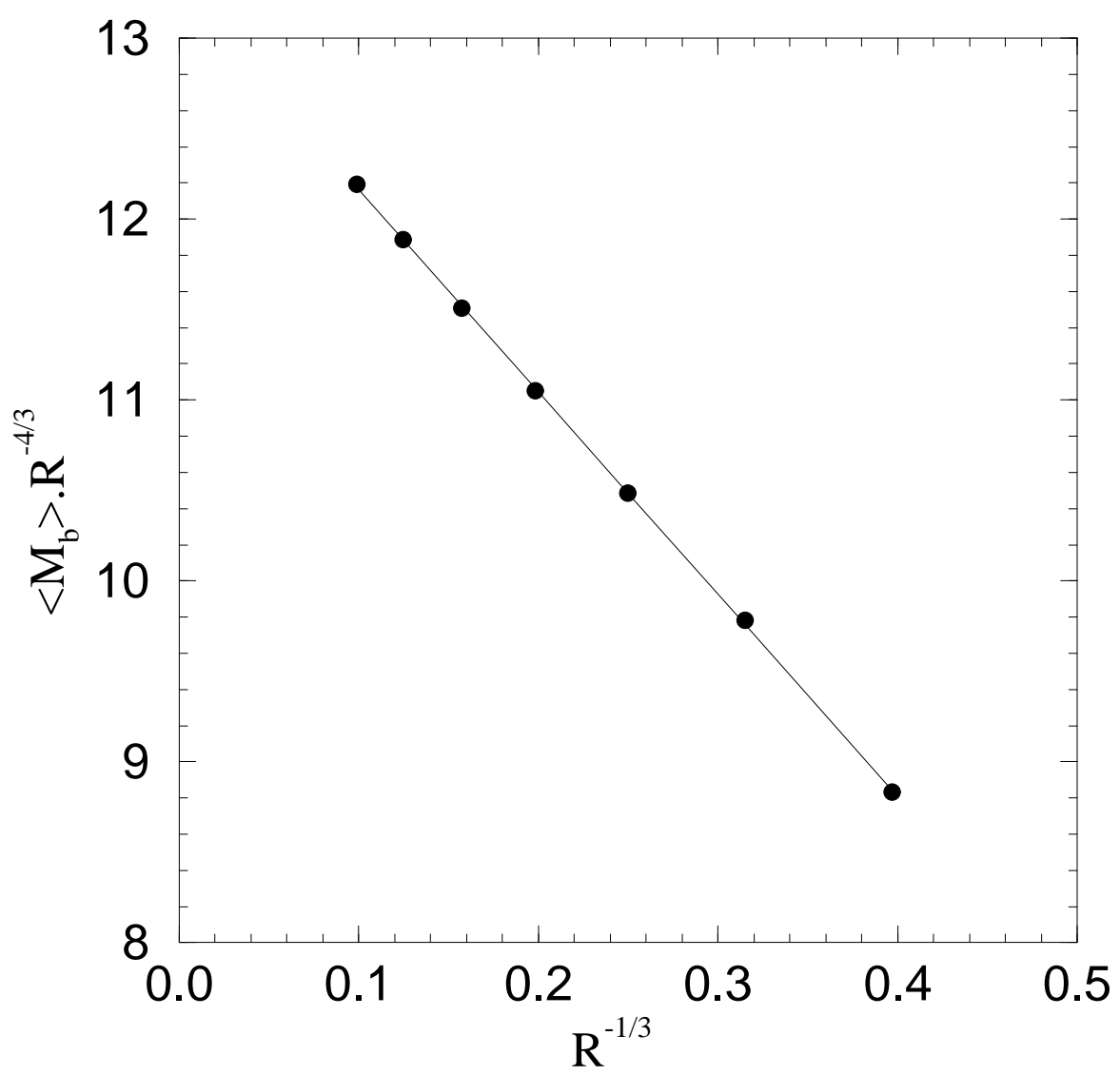




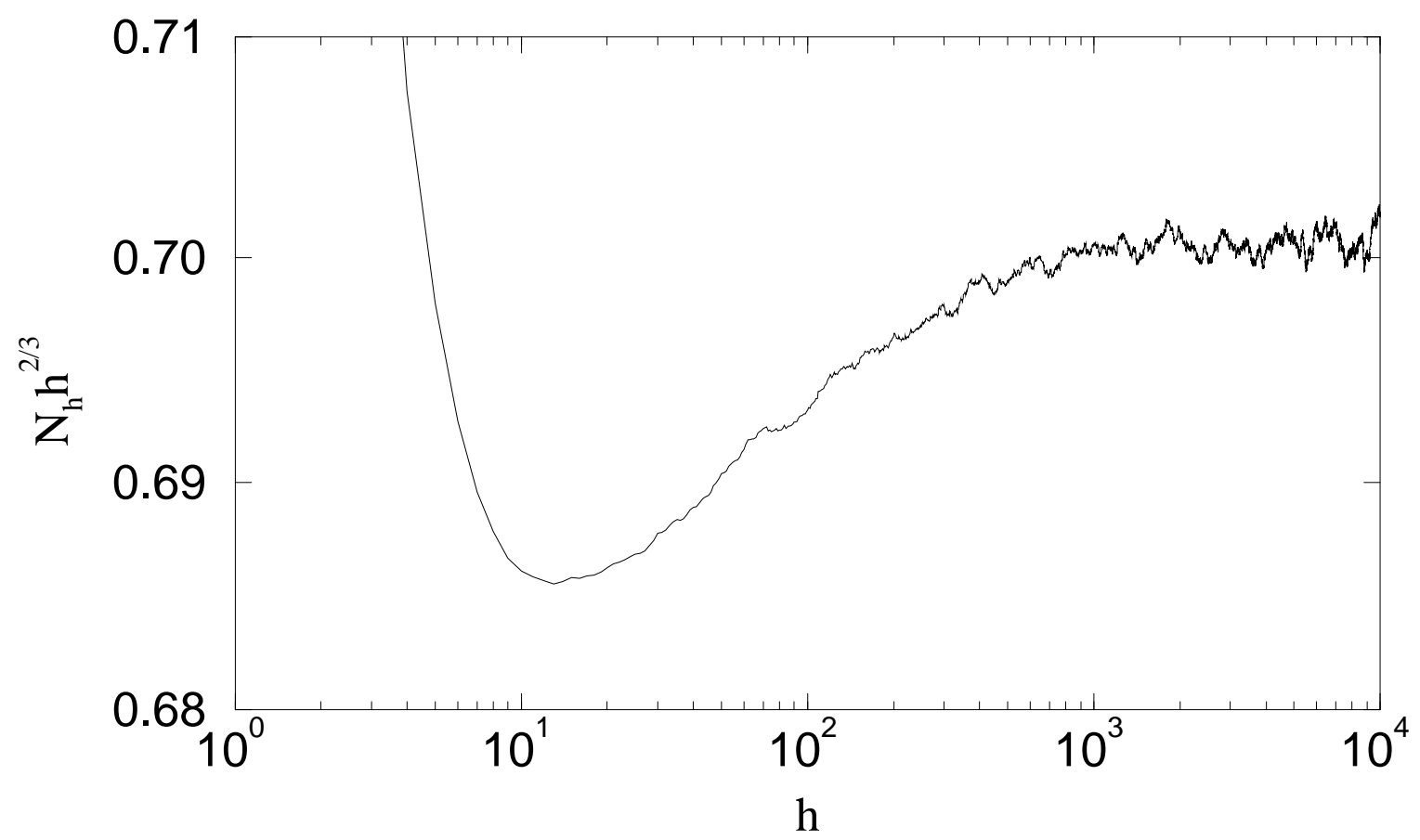




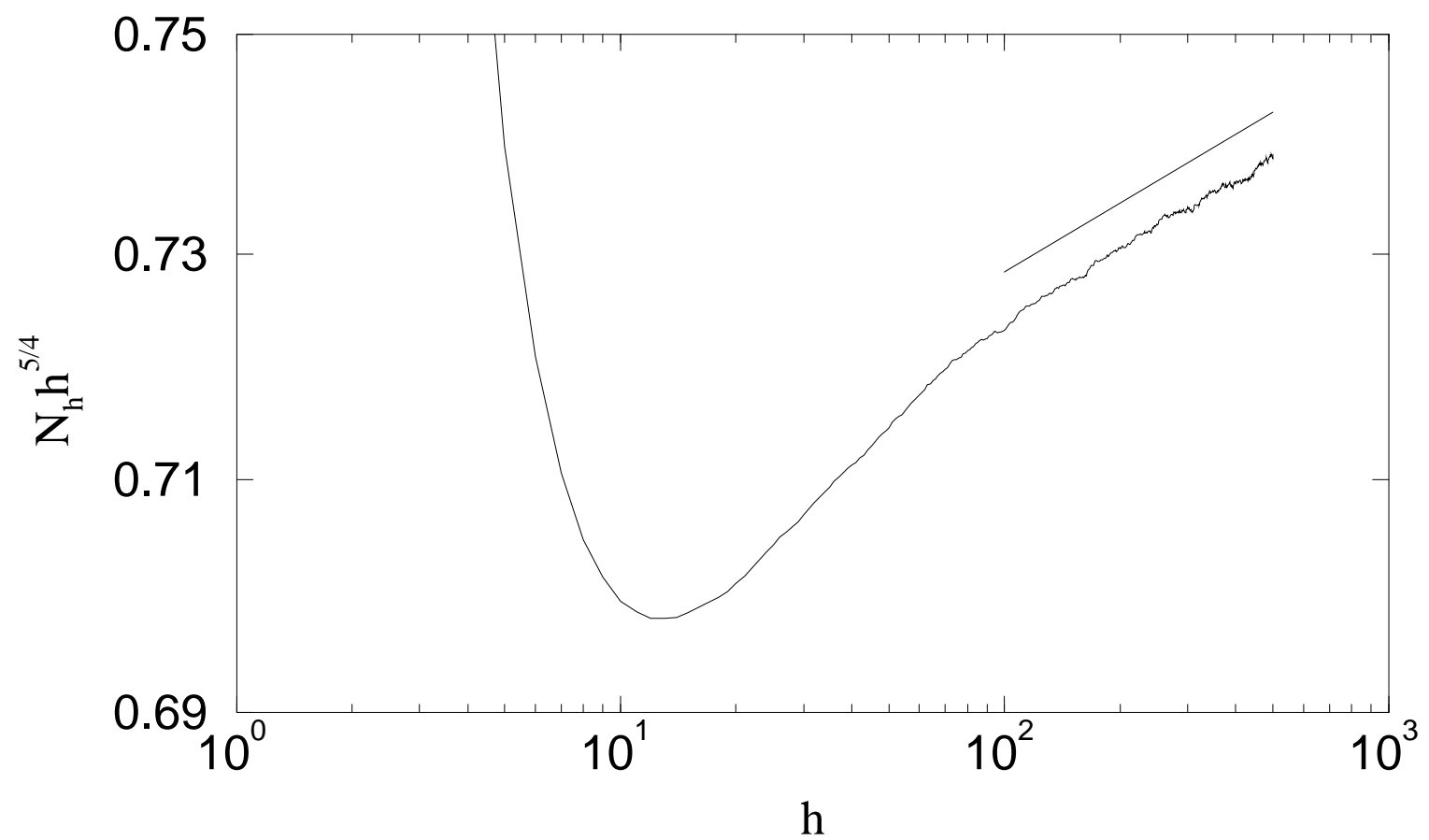

